\title{
Revisiting ADOPT-ing and its Feedback Schemes
}

\author{
Marius C. Silaghi \\ Florida Institute of Technology
}

\author{
Makoto Yokoo \\ Kyushu University
}

\begin{abstract}
Here we revisit ADOPT-ing and bring two new contributions. One contribution consists of developing variations on the algorithms keeping the improvement in length of chain of causal messages without an increase in the total number of messages. While past experiments have shown that sending more feedback is better than sending the minimal information needed for correctness, new experiments show that one should not exaggerate sending too much feedback and that the best strategy is at an intermediary point. This brings large efficiency improvements.

We also find that one of the nogood storages of ADOPTing can be removed without effects on efficiency while decreasing the space complexity by a factor given by the number of agents. We also provide a more general proof showing which types of nogood storages can be used in the inference of feedback without compromising correctness. In particular we show that all such structures can be updated by sum-inference, and from threshold messages.
\end{abstract}

\section{Introduction}

Distributed Constraint Optimization (DCOP) is a formalism that can model naturally distributed problems. These are problems where agents try to find assignments to a set of variables that are subject to constraints. Typically research has focused on techniques in which reluctance is manifested toward modifications to the distribution of the problem (modification accepted only when some reasoning infers it is unavoidable for guaranteeing that a solution can be reached). This criteria is widely believed to be valuable and adaptable for large, open, and/or dynamic distributed problems $[7,1,2,10]$. It is also perceived as an alternative approach to privacy requirements $[12,5,8]$.

Definition 1 (DCOP) A distributed constraint optimization problem (DCOP), is defined by a set of agents $A_{1}, A_{2}, \ldots, A_{n}$, a set $X$ of variables, $x_{1}, x_{2}, \ldots, x_{n}$, and a set of functions $f_{1}, f_{2}, \ldots f_{i}, \ldots, f_{n}, f_{i}: X_{i} \rightarrow \mathbb{R}_{+}$, $X_{i} \subseteq X$, where only $A_{i}$ knows $f_{i}$. We assume that $x_{i}$ can only take values from a domain $D_{i}=\{1, \ldots, d\}$.
Denoting with $x$ an assignment of values to all the variables in $X$, the problem is to find $\operatorname{argmin} \sum_{i=1}^{n} f_{i}\left(x_{\mid X_{i}}\right)$.

For simplification and without loss of generality, one typically assumes that $X_{i} \subseteq\left\{x_{1}, \ldots, x_{i}\right\}$.

By $x_{\mid X_{i}}$ we denote the projection the set of assignments in $x$ on the set of variables in $X_{i}$.

\section{ADOPT with nogoods}

Asynchronous Distributed OPTimization with inferences based on valued nogoods (ADOPT-ing) ${ }^{1}$ is a distributed optimization algorithm. It exploits the increased flexibility brought by the use of valued nogoods. The algorithm can be seen as an extension of both ADOPT [9] and asynchronous backtracking (ABT) [13].

A nogood, $\neg N$, specifies a set $N$ of assignments that conflict with existing constraints. Valued nogoods have the form $[S R C, c, N]$ and are an extension of classical nogoods. Each valued nogood has a set of references to a conflict list of constraints SRC and a threshold $c$. The threshold specifies the minimal weight of the constraints in the conflict list SRC given the assignments of the $\operatorname{nogood} N[3,11]$.

A valued nogood [ $\left.S R C, c, N \cup\left\langle x_{i}, v\right\rangle\right]$ applied to a value $v$ of a variable $x_{i}$ is referred to as the cost assessment (CA) of that value and is denoted $(S R C, v, c, N)$. If the conflict list is missing (and implies the whole problem) then we speak of a valued global nogood. One can combine valued nogoods by sum-inference and min-resolution to obtain new nogoods [3]. If $N=\left(\left\langle x_{1}, v_{1}\right\rangle, \ldots,\left\langle x_{t}, v_{t}\right\rangle\right)$ where $v_{i} \in D_{i}$, then we denote by $\bar{N}$ the set of variables assigned in $N$, $\bar{N}=\left\{x_{1}, \ldots, x_{t}\right\}$.

min-resolution: Assume that we have a set of cost assessments for $x_{i}$ of the form $\left(S R C_{v}, v, c_{v}, N_{v}\right)$ that has the property of containing exactly one CA for each value $v$ in the domain of variable $x_{i}$ and that for all $k$ and $j$, the assignments for variables $\overline{N_{k}} \cap \overline{N_{j}}$ are identical in both $N_{k}$ and $N_{j}$. Then the CAs in this set can be combined into a new valued nogood. The obtained valued nogood is $[S R C, c, N]$ such that $S R C=\cup_{i} S R C_{i}, c=\min _{i}\left(c_{i}\right)$ and $N=\cup_{i} N_{i}$.

\footnotetext{
${ }^{1}$ Introduced with the acronym ADOPT-ng in [11].
} 
sum-inference: A set of cost assessments of type $\left(S R C_{i}, v, c_{i}, N_{i}\right)$ for a value $v$ of some variable, where $\forall i, j: i \neq j \Rightarrow S R C_{i} \cap S R C_{j}=\emptyset$, and the assignment of any variable $x_{k}$ is identical in all $N_{i}$ where $x_{k}$ is present, can be combined into a new cost assessment. The obtained cost assessment is $(S R C, v, c, N)$ such that $S R C=\cup_{i} S R C_{i}, c=\sum_{i}\left(c_{i}\right)$, and $N=\cup_{i} N_{i}$.

As in ABT, agents communicate with ok? messages proposing new assignments of the variable of the sender, nogood messages announcing a nogood, and add-link messages announcing interest in a variable. As in ADOPT, agents can also use threshold messages, but their content can be included in ok? messages.

For simplicity we assume in this algorithm that the communication channels are FIFO. Attachment of counters to proposed assignments and nogoods also ensures this requirement (i.e., older assignments and older nogoods for the currently proposed value are discarded).

\subsection{Exploiting DFS trees for Feedback}

Here we recall the feedback schemes of ADOPT-ing and introduce the new variants ADOPT-A_- and ADOPT-D_.. In ADOPT-ing, agents are totally ordered as in ABT, $A_{1}$ having the highest priority and $A_{n}$ the lowest priority. The target of a valued nogood is the position of the lowest priority agent among those that proposed an assignment referred by that nogood. Note that the basic version of ADOPT-ing does not maintain a DFS tree, but each agent can send messages with valued nogoods to any predecessor. ADOPT-ing also has hybrid versions that can spare network bandwidth by exploiting an existing DFS tree. It has two ways of exploiting such an existing structure. The first is by having each agent send its valued nogood only to its parent in the tree and it is roughly equivalent to the original ADOPT. The other way is by sending valued nogoods only to ancestors. This later hybrid approach can be seen as a fulfillment of a direction of research suggested in [9], namely communication of costs to higher priority parents.

The versions of ADOPT-ing are differentiated using the notation ADOPT-XYZ. $\mathbf{X}$ shows the destinations of the messages containing valued nogoods. $\mathrm{X}$ has one of the values $\{p, a, A, d, D\}$ where $p$ stands for parent, $a$ and $A$ stand for all predecessors, and $d$ and $D$ stand for all ancestors in a DFS trees. Y marks the optimization criteria used by sum-inference in selecting a nogood when the inputs have the same threshold. For now we use a single criterion, denoted $o$, which consists of choosing the nogood whose target has the highest priority. $\mathbf{Z}$ specifies the type of nogoods employed and has possible values $\{n, s\}$, where $n$ specifies the use of valued global nogoods (without SRCs) and $s$ specifies the use of valued nogoods (with SRCs).

The different schemes are described in Figure 1. The to-

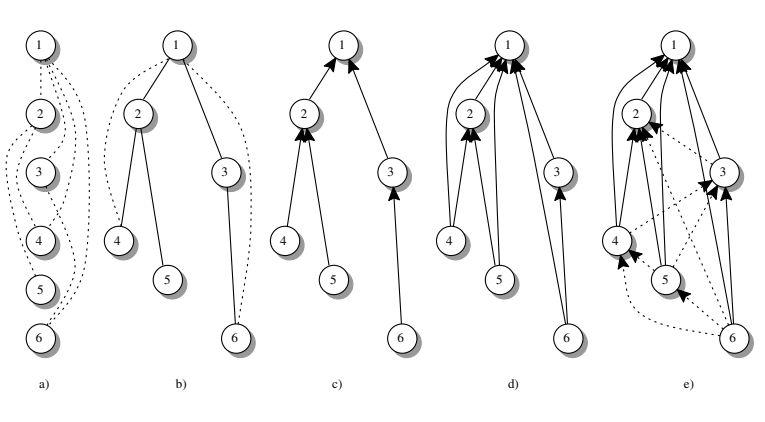

Figure 1. Feedback modes in ADOPT-ing.

tal order on agents is described in Figure 1.a where the constraint graph is also depicted with dotted lines representing the arcs. Each agent (representing its variable) is depicted with a circle. A DFS tree of the constraint graph which is compatible to this total order is depicted in Figure 1.b. ADOPT gets such a tree as input, and each agent sends COST messages (containing information roughly equivalent to a valued global nogood) only to its parent. As mentioned above, the versions of ADOPT-ing that replicate this behavior of ADOPT when a DFS tree is provided are called ADOPT- $\mathrm{p}_{\text {一, where }} \mathrm{p}$ stands for parent and the underscores stand for any legal value defined above for $\mathrm{Y}$ and $\mathrm{Z}$ respectively. This method of announcing conflicts based on the constraint graph is depicted in Figure 1.c and is related to the classic Graph-based Backjumping algorithm $[4,6]$.

In Figure 1.d we depict the nogoods exchange schemes used in ADOPT-d_ and ADOPT-D_- where, for each new piece of information, valued nogoods are separately computed to be sent to each of the ancestors in the known DFS tree. As for the initial version of ADOPT, the proof for ADOPT-d_ and ADOPT-D_ shows that the only mandatory nogood messages for guaranteeing optimality in this scheme are the ones to the parent agent. However, agents can infer from their constraints valued nogoods that are based solely on assignments made by shorter prefixes of the ordered list of ancestor agents. The agents try to infer and send valued nogoods separately for all such prefixes.

Figure 1.e depicts the basic versions of ADOPT-ing, when a DFS is not known (ADOPT-a_- and ADOPT-A_-), where nogoods can be sent to all predecessor agents. The dotted lines show messages, which are sent between independent branches of the DFS tree, and which are expected to be redundant. Experiments have shown that valued nogoods help to remove the redundant dependencies whose introduction would otherwise be expected from such messages. The provided proof for ADOPT-a_ and ADOPT-A_shows that the only mandatory nogood messages for guaranteeing optimality in this scheme are the ones to the immediately previous agent. However, agents can infer from their constraints valued nogoods that are based solely on as- 
signments made by shorter prefixes of the ordered list of all agents. As in the other case, the agents try to infer and send valued nogoods separately for all such prefixes.

A, D vs. a, d: The valued nogood computed for the prefix $A_{1}, \ldots, A_{k}$ ending at a given predecessor $A_{k}$ may not be different from the one of the immediately shorter prefix $A_{1}, \ldots, A_{k-1}$. Sending that nogood to $A_{k}$ may not affect the value choice of $A_{k}$, since the cost of that nogood applies equally to all values of $A_{k}$ (except when such nogoods cannot be composed by sum-inference with some valued nogoods of $A_{k}$.) The new versions ADOPT-D_- and ADOPTA_- correspond to the case where optional nogood messages are only sent when the target of the payload valued nogood is identical to the destination of the message. The versions ADOPT-d_- and ADOPT-a_- correspond to the case where optional nogood messages are sent to all possible destinations each time that the payload nogood has a non-zero threshold. I.e., in those versions nogood messages are sent even when the target of the transported nogood is not identical to the destination agent but has a higher priority.

\subsection{Data Structures}

Each agent $A_{i}$ stores its agent-view (received assignments), and its outgoing links (agents of lower priority than $A_{i}$ and having constraints on $x_{i}$ ). The instantiation of each variable is tagged with the value of a separate counter incremented each time the assignment changes. To manage nogoods and CAs, $A_{i}$ uses matrices $l[1 . . \mathrm{d}], h[1 . . \mathrm{d}], c a[1 . . \mathrm{d}][i+1 . . \mathrm{n}], \operatorname{th}[1 . \mathrm{i}], \operatorname{lr}[\mathrm{i}+1 . . \mathrm{n}]$ and lastSent[1..i-1] where $d$ is the domain size for $x_{i}$. crt_val is the current value $A_{i}$ proposes for $x_{i}$.

- $l[\mathrm{k}]$ stores a CA for $x_{i}=k$, inferred solely from the local constraints between $x_{i}$ and prior variables.

- $c a[\mathrm{k}][\mathrm{j}]$ stores a CA for $x_{i}=k$, which is obtained by sum-inference from valued nogoods received from $A_{j}$.

- $t h[\mathrm{k}]$ stores nogoods coming via threshold/ok? messages from $A_{k}$.

- $h[\mathrm{v}]$ stores a CA for $x_{i}=\mathrm{v}$, which is inferred from $c a[\mathrm{v}][\mathrm{j}], l[\mathrm{v}]$ and $t h[\mathrm{t}]$ for all $t$ and $j$.

- $\operatorname{lr}[\mathrm{k}]$ stores the last valued nogood received from $A_{k}$.

- lastSent[k] stores the last valued nogood sent to $A_{k}$.

The names of the structures follow the relation of ADOPT with A* search. Thus, $h$ stands for the "heuristic" estimation of the cost due to constraints maintained by future agents (equivalent to the $h()$ function in $\mathrm{A}^{*}$ ) and $l$ stands for the part of the standard $g()$ function of $\mathrm{A}^{*}$ that is "local" to the current agent. Here, as in ADOPT, the value for $h()$ is estimated by aggregating the equivalent of costs received from lower priority agents. Since the costs due to constraints of higher priority agents are identical for each value, they are irrelevant for the decisions of the current agent. Thus, the function $f()$ of this version of $\mathrm{A}^{*}$ is computed combining solely $l$ and $h$. We currently store the result of combining $h$ and $l$ in $h$ itself to avoid allocating a new structure for $f()$.

The structures $l r$ and $t h$ store received valued nogoods and $c a$ stores intermediary valued nogoods used in computing $h$. The reason for storing $l r, t h$ and $c a$ is that change of context may invalidate some of the nogoods in $h$ while not invalidating each of the intermediary components from which $h$ is computed. Storing these components (which is optional) saves some work and offers better initial heuristic estimations after a change of context. The cost assessments stored in $c a[v][j]$ of $A_{i}$ also maintain the information needed for threshold messages, namely the heuristic estimate for the value $v$ of the variable $x_{i}$ at successor $A_{j}$ (to be transmitted to $A_{j}$ if the value $v$ is proposed again).

The array lastSent is used to store at each index $k$ the last valued nogood sent to the agent $A_{k}$. The array $l r$ is used to store at each index $k$ the last valued nogood received from the agent $A_{k}$. Storing them separately guarantees that in case of changes in context, they are discarded at the recipient only if they are also discarded at the sender. This property guarantees that an agent can safely avoid retransmitting to $A_{k}$ messages duplicating the last sent nogood, since if it has not yet been discarded from lastSent $[k]$ then the recipients have not discarded it from $\operatorname{lr}[k]$ either.

\subsection{Data flow in ADOPT-ing}

The pseudocode is described in Algorithm 1. The min_resolution $(j)$ function applies the min-resolution over the CAs associated to all the values of the variable of the current agent, but uses only CAs having no assignment from agents with lower priority than $A_{j}$. More exactly it first re-computes the array $h$ using only CAs in $c a$ and $l$ that contain only assignments from $A_{1}, \ldots, A_{j}$, and then applies min-resolution over the obtained elements of $h$. As mentioned above, in the current implementation we recompute $l$ and $h$ at each call to min_resolution $(j)$, and such a call is separately performed for each ancestor agent $A_{j}$.

The order of combining CAs matters. To compute $h[\mathrm{v}]$ :

1. a) When maintaining DFS trees, for each value $v$, CAs are combined separately for each set $s$ of agents defining a DFS sub-tree of the current node: $\operatorname{tmp}[\mathrm{v}][s]=$ sum-inference $t_{t \in s}(\mathrm{ca}[\mathrm{v}][\mathrm{t}])$.

b) Otherwise, with ADOPT-a_ and ADOPT-A_-, we act as if we have a single sub-tree: $\operatorname{tmp}[\mathrm{v}]=$ sum-inference $_{t \in[i+1, n]}(\mathrm{ca}[\mathrm{v}][\mathrm{t}])$.

2. CAs from step 1 (a or b) are combined: In case (a) this means: 
when receive ok? $\left(\left\langle x_{j}, v_{j}\right\rangle\right.$, tvn $)$ do

// tvn is a threshold valued nogood

integrate $\left(\left\langle x_{j}, v_{j}\right\rangle\right)$;

if (tvn no-null and has no old assignment) then

k:=target $(t v n)$; // threshold tvn as common cost;

L $t h[\mathrm{k}]:=$ sum-inference $(t v n, t h[\mathrm{k}])$;

check-agent-view();

when receive add-link $\left(\left\langle x_{j}, v_{j}\right\rangle\right)$ from $A_{j}$ do

add $A_{j}$ to outgoing-links;

if $\left(\left\langle x_{j}, v_{j}\right\rangle\right)$ is old, send new assignment to $A_{j}$;

when receive $\operatorname{nogood}(r v n, t)$ from $A_{t}$ do

foreach new assignment a of a linked variable $x_{j}$ in rvn do

$\mathrm{L}$ integrate $(a)$; // counters show newer assignment;

if (an assignment in rvn is outdated) then

if (some new assignment was integrated now) then

$\mathrm{L}$ check-agent-view();

return;

foreach assignment a of a non-linked variable $x_{j}$ in rvn do $\mathrm{L}$ send add-link(a) to $A_{j}$;

$\operatorname{lr}[\mathrm{t}]:=r v n$;

foreach value $v$ of $x_{i}$ such that $r v n_{\mid v}$ is not $\emptyset \mathbf{d o}$ $v n 2 c a(r v n, i, v) \rightarrow r c a\left(\right.$ a CA for the value $\mathrm{v}$ of $\left.x_{i}\right)$; $c a[\mathrm{v}][\mathrm{t}]:=$ sum-inference $(r c a, c a[\mathrm{v}][\mathrm{t}])$;

update $h[\mathrm{v}]$ and retract changes to $c a[\mathrm{v}][\mathrm{t}]$

if $h[\mathrm{v}]$ 's cost decreases;

check-agent-view();

procedure check-agent-view() do

for every $A_{j}$ with higher priority than $A_{i}$ (respectively ancestor in the DFS tree, when one is maintained) do

for every $\left(v \in D_{i}\right)$ update $l[v]$ and recompute $h[v]$; // with valued nogoods using only instantiations // of $\left\{x_{1}, \ldots, x_{j}\right\}$;

if ( $h$ has non-null cost $C A$ for all values of $D_{i}$ ) then

vn:=min_resolution(j);

if $(v n \neq$ lastSent $[j])$ then

if $(\operatorname{target}(v n)==j)$ then

send $\operatorname{nogood}(v n, i)$ to $A_{j}$;

lastSent $[\mathrm{j}]=\mathrm{vn}$;

$c_{\text {crt_val }} \operatorname{argmin}_{v}(\operatorname{cost}(h[v]))$;

if (crt_val changed) then

send ok? $\left(\left\langle x_{i}, c r t_{-} v a l\right\rangle, \mathrm{ca} 2 \mathrm{vn}(c a[\right.$ crt_val $\left.][\mathrm{k}]), i\right)$

to each $A_{k}$ in outgoing_links;

procedure integrate $\left(\left\langle x_{j}, v_{j}\right\rangle\right)$ do

discard elements in $c a$, th, lastSent and $l r$ based on other values for $x_{j}$;

use $\operatorname{lr}[\mathrm{t}]_{\mid v}$ to replace each discarded $c a[\mathrm{v}][\mathrm{t}]$;

store $\left\langle x_{j}, v_{j}\right\rangle$ in agent-view;

procedure init do

$h[v]:=l[v]:=$ initialize CAs from unary constraints;

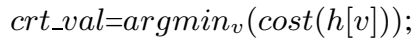

send ok? $\left(\left\langle x_{i}, c r t \_v a l\right\rangle, \emptyset\right)$ to all agents in outgoing-links;

Algorithm 1: Receiving messages of $A_{i}$ in ADOPT-ing $\forall v, s ; h[v]=$ sum-inference $_{\forall s}(\operatorname{tmp}[\mathrm{v}][\mathrm{s}])$.

Note that the SRCs in each term of this sum-inference are disjoint and therefore we obtain a valued nogood with threshold given by the sum of the individual thresholds obtained for each DFS sub-tree (or larger).

For case (b) we obtain $\mathrm{h}[\mathrm{v}]=\mathrm{tmp}[\mathrm{v}]$. This makes sure that at quiescence the threshold of $h[v]$ is at least equal to the total cost obtained at the next agent.

3. Add $1[\mathrm{v}]: \mathrm{h}[\mathrm{v}]=$ sum-inference $(\mathrm{h}[\mathrm{v}], \mathrm{l}[\mathrm{v}])$.

4. Add threshold: $\mathrm{h}[\mathrm{v}]=$ sum-inference $(\mathrm{h}[\mathrm{v}], \mathrm{th}[*])$.

The following is a new result:

Lemma 1 (Infinite Cycle) At a given agent, assume that the agent-view no longer changes and that its array $h$ (used for min-resolution and for deciding the next assignment) is computed only using cost assessments that are updated solely by sum-inference. In this case the thresholds of the elements of its $h$ cannot be modified in an infinite cycle.

Proof. Valued nogoods that are updated solely by suminference have thresholds that can only increase (which can happen only a finite number of times). For a given threshold, modifications can only consist of modifying assignments to obtain lower target agents, which again can happen only a finite number of times. Therefore, after a finite number of events, the cost assessments used to infer $h$ will not be modified any longer and therefore $h$ will no longer be modified.

Corollary 1.1 If ADOPT-ing uses the aforementioned procedure, then for a given agent-view, the elements of the array $h$ for that agent cannot be modified in an infinite cycle.

Remark 1 Since lr contains the last received valued nogoods via messages other than ok? messages which change the agent-view, that array is updated by assignment with recently received nogoods without sum-inference. Therefore, we do not use it directly to infer $h$. We use lr only to initialize ca after a change of the agent-view.

Note that with the described procedure, a newly arriving valued nogood can decrease the threshold of certain elements of $h$. This is because, while increasing the threshold of some element in $c a$, it can also modify its SRC and therefore forbid its composition by sum-inference with other cost assessments.

Remark 2 (Obtaining Monotonic Increase) The implementation used for the experiments reported here avoids the undesired aforementioned effect, where incoming nogoods decrease thresholds of cost assessments in h. Namely, after 
a newly received valued nogood is added by sum-inference to the corresponding element of $c a[v]$ for some value $v$, if the threshold of $h[v]$ decreases then the old content of $c a[v]$ is restored. Each new valued nogood is used for updating lr. However, on each change of the agent-view (set of known valid assignments), all values of ca are updated using the valued nogoods found in $l r$ and th.

Lemma 2 Revised ADOPT-ing terminates in finite time.

Proof.

Given the list of agents $A_{1}, \ldots, A_{n}$, define the suffix of length $m$ of this list as the last $m$ agents. Then the result follows immediately by induction for an increasingly growing suffix (increasing $m$ ), assuming the other agents reach quiescence.

The basic case of the induction (for the last agent) follows from the fact that the last agent terminates in one step if the previous agents do not change their assignments.

Let us now assume that the induction assertion is true for a suffix of $k$ agents. Based on this assumption we now prove the induction step, namely that the property is also true for a suffix of $k+1$ agents: For each assignment of the agent $A_{n-k}$, the remaining $k$ agents will reach quiescence, according to the assumption of the induction step; otherwise, the assignment's CA threshold increases. By construction, thresholds for CAs associated with the values of $A_{n-k}$ can only grow (see Remark 2). Even without the technique in Remark 2, thresholds for CAs associated with the values of $A_{n-k}$ will eventually stop being modified as a consequence of Lemma 1. After values are proposed in turn and the smallest threshold reaches its highest estimate, agent $A_{n-k}$ selects the best value and reaches quiescence. The other agents reach quiescence according to the induction step.

The following results were proven in [11] and remain true for the described generalization.

Lemma 3 The last valued nogoods sent by each agent additively integrate the non-zero costs of the constraints of all of the agent's successors.

\section{Theorem 4 ADOPT-ing returns an optimal solution.}

Note that the lvn data structure mentioned in [11] is no longer used. That data structure holds $n^{2}$ valued nogoods and its removal has little impact on the experimental results. Since remaining arrays have dimensions that are only linear in $n$, the space complexity of the revisited version is smaller than the one of the original version by the factor $n$.

\subsection{Example}

Now we give a detailed example of a run of ADOPT-ing basic versions ADOPT-aos and ADOPT-Aos. Let us take

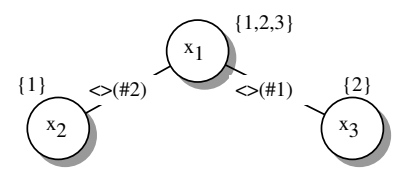

\section{Figure 2. The penalty of conflicting the con- straint $x_{1} \neq x_{2}$ is $\mathbf{2}$, denoted (\#2).}

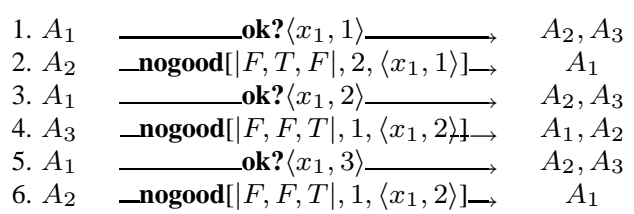

Figure 3. Trace of ADOPT-aos and ADOPTAos on the problem in Figure 2

the problem in Figure 2. Note that in this simple case the two versions do not differ since any optional nogood message can only leave from $A_{3}$ to $A_{1}$. Such a message is sent in ADOPT-aos only if it has a non-zero threshold, which happens only when $A_{1}$ is a target of the message, which means that it will also be sent in ADOPT-Aos. A trace is shown in Figure 3 where identical messages sent simultaneously to several agents are grouped by displaying the list of recipients. The agents start selecting values for their variables and announce them to interested lower priority agents. $A_{3}$ has no constraint between $x_{3}$ and $x_{2}$; therefore the first exchanged messages are ok? messages sent by $A_{1}$ to both successors $A_{2}$ and $A_{3}$ and proposing the assignment $x_{1}=1$.

After receiving the assignment from $A_{1}$, the best (and only) assignment for $A_{2}$ is $x_{2}=1$ at a cost of 2 due to the conflict with the constraint $x_{1} \neq x_{2}$. Similarly $A_{3}$ instantiates $x_{3}$ with 2 and with a local cost of 0 .

Since the best local cost of $A_{2}$ is not null, $A_{2}$ performs a min-resolution. Since a single value exists for $A_{2}$ and $c a$ is empty, this min-resolution simply obtains a valued nogood defined by the existing local nogood: $h[1]=l[1]=$ $\left[C_{1,2}, 2,\left\langle x_{1}, 1\right\rangle\right]$. In our implementation we decide to maintain a single reference for each agent's secret constraints. SRCs are represented as Boolean values in an array of size $n$. A value on the $i^{\text {th }}$ position in the array SRC equal to $T$ signifies that the constraints of $A_{i}$ are used in the inference of that nogood. $A_{2}$ also stores the sent valued nogood in lastSent [1] such that it avoids resending it without modification as a result of receiving other messages. $A_{1}$ stores this received valued nogood in $\operatorname{lr}[2]$, from where it is used to update $c a[1][2]$, by sum-inference. Since $c a[1][2]$ is empty, it becomes equal to this valued nogood.

Agent $A_{1}$ now updates its $h[1]$ by setting it to $c a[1][2]$ (since $l[1]$ and $c a[1][3]$ are empty). Since the threshold of 


\begin{tabular}{|c|c|c|c|c|c|}
\hline Agents & ADOPT & aos & Aos & dos & Dos \\
\hline 14 & 75.64 & 42.32 & 42.8 & 42.44 & 42.72 \\
25 & 221.44 & 83.12 & 83.96 & 80.64 & 84.2 \\
30 & 433.92 & 112.68 & 122.64 & 112.52 & 114.84 \\
40 & 720.04 & 117.28 & 108.4 & 107.64 & 112.24 \\
\hline
\end{tabular}

Table 1. Cycles at density $20 \%$.

$h[1]$ becomes 2 and is higher than the threshold of the other two values, $\{2,3\}$, in the domain of $x_{1}, A_{1}$ changes the assignment of $x_{1}$ to one of them, here 2. This is announced through another ok? message to $A_{2}$ and $A_{3}$.

On the receipt of the ok? messages, the agents update their agent-view with the new assignment. Each agent tries to generate valued nogoods for each prefix of its list of predecessor agents: $\left\{A_{1}\right\}$ and $\left\{A_{1}, A_{2}\right\}$ respectively. This time it is $A_{3}$ whose only possible assignment leads to a nonzero local cost. Based on its agent-view and constraints, $A_{3}$ generates a corresponding valued nogood $\left[C_{1,3}, 1,\left\langle x_{1}, 2\right\rangle\right]$ with threshold 1 due to the weight 1 of its constraint. This valued nogood is sent to the agent $A_{1}$ whose assignment is involved in this nogood. To guarantee optimality the nogood is also sent to its immediate predecessor, namely the agent $A_{2}$, making sure that at quiescence all the costs of its children are summed.

After receiving this second nogood, $A_{1}$ stores it in $\operatorname{lr}[3]$, used further by sum-inference to set $c a[2][3]$, and finally used to update $h[2]$. As a result, $A_{1}$ now switches its assignment to its value that has the lowest threshold in $h$, namely the value 3 . The new assignment is again sent by ok? messages to its successors. Meanwhile, the agent $A_{2}$ also processes the valued nogood received from $A_{3}$ storing it in its own $l r[3], c a[2][3]$ and $h[2]$. The nogood is not changed by sum inference or min-resolution at this agent; it is sent on to $A_{1}$ which stores it in $\operatorname{lr}[2]$ and $c a[2][2]$. However, it does not lead to any modification in the $h[2]$ of $A_{1}$ since the SRCs of $c a[2][2]$ and $c a[2][3]$ have a nonempty intersection.

After receiving the third assignment from $A_{1}$, the other two agents reach quiescence with cost 0 ; thus an optimal solution is found. Note that the existence of message 6 depends on whether the message 5 (with the last assignment from $A_{1}$ ) reaches $A_{2}$ before or after the nogood from $A_{3}$, that the message 5 invalidates. The solution is found in 5 half-round-trips of messages (a logic time of 5).

\section{Experiments}

The algorithms are compared on the same problems that are used to report ADOPT's performance in [9]. To correctly compare our techniques with the original ADOPT and ADOPT-ing, we have used the same order (or DFS trees) on agents for each problem. The impact of the existence of a good DFS tree compatible with the used order

\begin{tabular}{|c|c|c|c|c|c|}
\hline Nodes & aos & Aos & dos & Dos & pon \\
\hline 14 & 21.981 & 14.696 & 15.760 & 12.427 & 16.869 \\
25 & 863.14 & 350.33 & 602.43 & 291.92 & 630.51 \\
30 & 3640.81 & 1137.31 & 1853.42 & 881.04 & 830.61 \\
40 & 49803 & 9046.12 & 22414 & 7141.7 & \\
\hline
\end{tabular}

Table 2. Thousands messages at density .3.

is tested separately by comparison with a random ordering. The set of problems distributed with ADOPT and used here contains 25 problems for each problem size. It contains problems with up to 40 agents, and it contains test sets with density .2 and with density .3 .

The length of the longest causal (sequential) chain of messages of each solver, computed as the number of cycles of our simulator is given in Tables 3 and 1. It took more than two weeks for the original ADOPT implementation to solve one of the problems for 20 agents and density .3, and one of the problems for 25 agents and density .3 (at which moment the solver was interrupted).

The use of valued nogoods in ADOPT-ing brought an improvement of approximately 7 times on problems of density 0.2 , and an approximately 5 times improvement on the problems of density .3 .

Table 3 shows that, with respect to the number of cycles, the use of SRCs practically replaces the need to maintain the DFS tree since ADOPT-aos and ADOPT-Aos are comparable in efficiency with ADOPT-dos and ADOPT-Dos. SRCs bring improvements over versions with valued global nogoods, since SRCs allow detection of dynamically obtained independence. Versions using DFS trees require fewer total messages, being more network friendly, as seen in Table 2. Table 2 shows that refraining from sending too many optional nogoods messages, as done in ADOPT-Aos and ADOPT-Dos, is comparable to ADOPT-pon in terms of total number of messages, while maintaining the efficiency in cycles comparable to ADOPT-aos and ADOPT-dos.

Another experiment, whose results are shown in Table 4, is meant to evaluate the impact of the guarantees that the ordering on agents is compatible with some short DFS tree. We evaluate this by comparing ADOPT-aos with an ordering that is compatible with the DFS tree built by ADOPT, versus a random ordering. The results show that random orderings are unlikely to be compatible with short DFS trees and that verifying the existence of a short DFS tree compatible to the ordering on agents to be used by ADOPT-ing is highly recommended.

Table 3 clearly show that the highest improvement in number of cycles is brought by sending valued nogoods to other ancestors besides the parent. The use of the structures of the DFS tree makes slight improvements in number of cycles (when nogoods reach all ancestors) and slight improvements in total message exchange. To obtain a low total 


\begin{tabular}{|c|c|c|c|c|c|}
\hline Agents & ADOPT & aos & Aos & dos & Dos \\
\hline 14 & 1591 & 674.56 & 704.96 & 656.24 & 669.44 \\
18 & 4666.4 & 1777.44 & 1815.6 & 1727.84 & 1765.16 \\
30 & $* 58459.1$ & 16707.48 & 17618.48 & 16097.36 & 17154.4 \\
40 & $*$ & 96406.76 & 90747.6 & 93678.76 & 90951.56 \\
\hline
\end{tabular}

Table 3. Cycles at density .3. Entries with * show algorithm had to be interrupted.

\begin{tabular}{|c|c|c|c|c|c|c|}
\hline Agents & 16 & 18 & 20 & 25 & 30 & 40 \\
\hline $\begin{array}{c}\text { DFS compatible } \\
\text { random order }\end{array}$ & 839.92 & 1777.44 & 1711.84 & 7499.32 & $16^{*} 10^{3}$ & $96^{*} 10^{3}$ \\
\hline
\end{tabular}

Table 4. Impact of choice of order at density .3 .

message traffic and to reduce computation at agent level, we found that it is best not to announce any possible valued nogoods to each interested ancestor. Instead, one can reduce the communication without a penalty in number of cycles by only announcing valued nogoods to the highest priority agent to which they are relevant (besides the communication with the parent, required for guaranteeing optimality).

\section{Conclusions}

The Asynchronous Distributed Optimization with nogoods (ADOPT-ing) algorithm is revisited and its space complexity is reduced by one factor without a negative impact on efficiency. Its proof is generalized to allow for extensions of its nogood storage schemes. Previous experimentation with ADOPT-ing has shown that it is important for an agent to infer and send in parallel several valued nogoods to different higher priority agents [11]. That conclusion was drawn by experimenting with the two extreme settings: 1) where no optional message with valued nogoods is sent (similar to ADOPT), and 2) where any inferred valued nogood is sent to all ancestors able to handle it. The first extreme was found to be an order of magnitude better than the other extreme in terms of total number of messages. The second extreme is an order of magnitude better than the first one in length of the longest causal chain of messages.

Experiments show that an intermediary setting has the advantages of both extremes. If each inferred valued nogood is sent only to the highest priority agent that can handle it (its target), than the protocol is comparable to ADOPT in the total number of messages and comparable to the other extreme in length of longest causal chain of messages.

We determined the importance of precomputing and maintaining a short DFS tree of the constraint graph, or at least of guaranteeing that a DFS tree is compatible with the order on agents, which is almost an order of magnitude in our problems. Choosing a strategy of medium aggressiveness for sending valued nogoods to predecessors brings slight improvements in terms of length of the longest causal chain of messages (measured as number of cycles of the simulator). It brings an order of magnitude improvements in the total number of messages, as in ADOPT.

\section{References}

[1] S. Ali, S. Koenig, and M. Tambe. Preprocessing techniques for accelerating the DCOP algorithm ADOPT. In AAMAS, 2005.

[2] A. Chechetka and K. Sycara. No-commitment branch and bound search for distributed constraint optimization. In $A A$ MAS, 2006.

[3] P. Dago and G. Verfaillie. Nogood recording for valued constraint satisfaction problems. In ICTAI, 1996.

[4] R. Dechter. Enhancement schemes for constraint processing: Backjumping, learning, and cutset decomposition. AI'90, 1990.

[5] R. Greenstadt, J. Pearce, E. Bowring, and M. Tambe. Experimental analysis of privacy loss in dcop algorithms. In AAMAS, pages 1024-1027, 2006.

[6] Y. Hamadi and C. Bessière. Backtracking in distributed constraint networks. In ECAI'98, pages 219-223, 1998.

[7] R. Maheswaran, M. Tambe, E. Bowring, J. Pearce, and P. Varakantham. Taking DCOP to the real world: Efficient complete solutions for distributed event scheduling. In $A A$ MAS, 2004.

[8] R. Mailler and V. Lesser. Solving distributed constraint optimization problems using cooperative mediation. In $A A M A S$, pages 438-445, 2004.

[9] P. J. Modi, W.-M. Shen, M. Tambe, and M. Yokoo. ADOPT: Asynchronous distributed constraint optimization with quality guarantees. AIJ, 161, 2005.

[10] A. Petcu and B. Faltings. ODPOP: an algorithm for open/distributed constraint optimization. In AAAI, 2006.

[11] M.-C. Silaghi and M. Yokoo. Nogood-based asynchronous distributed optimization. In AAMAS, 2006.

[12] R. Wallace and E. Freuder. Constraint-based multi-agent meeting scheduling: Effects of agent heterogeneity on performance and privacy loss. In DCR, pages 176-182, 2002.

[13] M. Yokoo, E. H. Durfee, T. Ishida, and K. Kuwabara. The distributed constraint satisfaction problem: Formalization and algorithms. IEEE TKDE, 10(5):673-685, 1998. 